\title{
Coexistence of dipolar frustration and criticality in ferromagnets
}

\author{
Sergio A. Cannas ${ }^{1, *}$ and Alessandro Vindigni ${ }^{2, \dagger}$ \\ ${ }^{1}$ Facultad de Matemática, Astronomía, Física y Computación, Universidad Nacional de Córdoba and Instituto de Física Enrique Gaviola \\ (IFEG-CONICET), Ciudad Universitaria, 5000 Córdoba, Argentina \\ ${ }^{2}$ Laboratorium für Festkörperphysik, Eidgenössische Technische Hochschule Zürich, CH-8093 Zürich, Switzerland
}

(Received 15 June 2018; revised manuscript received 29 October 2018; published 20 December 2018)

\begin{abstract}
In real magnets the tendency toward ferromagnetism — promoted by exchange coupling —is usually frustrated by dipolar interaction. As a result, the uniformly ordered phase is replaced by modulated (multidomain) phases, characterized by different order parameters rather than the global magnetization. The transitions occurring within those modulated phases and toward the disordered phase are generally not of second-order type. Nevertheless, strong experimental evidence indicates that a standard critical behavior is recovered when comparatively small fields are applied that stabilize the uniform phase. The resulting power laws are observed with respect to a putative critical point that falls in the portion of the phase diagram occupied by modulated phases, in line with an avoided-criticality scenario. Here we propose a generalization of the scaling hypothesis for ferromagnets, which explains this observation assuming that the dipolar interaction acts as a relevant field, in the sense of renormalization group. We corroborate this proposal with analytic and numerical calculations on the 2D Ising model frustrated by dipolar interaction (isotropic part). Our analysis is directly applicable to thin magnetic films with out-of-plane anisotropy.
\end{abstract}

DOI: 10.1103/PhysRevE.98.062131

\section{INTRODUCTION}

The continuous comparison with model experimental systems has played a crucial role in the development of the theory of cooperative phenomena. In particular, magnetic systems have been a suitable playground for the study of secondorder phase transitions. In correspondence to a second-order phase transition observables follow a power-law behavior as a function of external parameters. Such a power-law behavior defines the condition of criticality. The property that different physical systems may follow the same power laws in the vicinity of the respective critical points is referred to as universality of critical exponents [1,2]. Celebrated models that successfully reproduce this universal aspect of secondorder phase transitions are (normally) based on short-ranged interactions [2-4]. When applied to the ferromagnetic-toparamagnetic phase transition, these textbook cooperative models are compatible with low-temperature magnetization curves at thermodynamic equilibrium similar to the discontinuous curve in Fig. 1. The singularity in the magnetization curve originates from the very same nonanalyticity that explains criticality and universality of critical exponents. In practical cases, the magnetization as a function of the external field $B$ does not jump from one branch to the other when $B=0$ is crossed: the system rather remains in a metastable configuration and the curve displays magnetic hysteresis, a typical out-of-equilibrium phenomenon (Fig. 1). In real magnets the short-ranged exchange interaction, which drives the establishment of ferromagnetism at low temperature, coexists

\footnotetext{
*cannas@famaf.unc.edu.ar

${ }^{\dagger}$ vindigni@phys.ethz.ch
}

with the long-ranged dipolar interaction. This second interaction generally frustrates the realization of a phase with uniform magnetization throughout a sample, consistent with the Griffiths' theorem [5,6] for bulk magnets. The compromise most often encountered in experiments is the occurrence of a multidomain phase (highlighted by the ellipse in Fig. 1). The discontinuity marked in Fig. 1 with two bullets on the equilibrium magnetization curve produced by models with short-ranged interactions only is replaced by an analytic function when dipolar interaction is taken into account.

A fundamental question then arises: along with the discontinuity in the equilibrium magnetization curve does dipolar interaction wipes away the critical behavior as well? In the following we consider a minimal model and show that the dipolar interaction acts as a relevant field, in the meaning of the renormalization group, beside the reduced temperature and the external $B$ field [2,7]. This description is able to account for the coexistence of ordinary criticality with an analytic behavior of the magnetization as a function of $B$ at every temperature.

In Sec. II, we introduce the model and summurize the main experimental facts that inspired our study. In Sec. III, we propose a scaling ansatz that is validated in the forthcoming sections with a mean-field calculation (Sec. IV), a real-space renormalization group approach (Sec. V), and Monte Carlo (MC) simulations (Sec. VI).

\section{EXPERIMENTAL FACTS AND THE MODEL}

In this paper, we address properties related to thermodynamic equilibrium with particular focus on the behavior of the magnetization as a function of the applied field $B$. Magnetic hysteresis, usually considered the distinctive feature of the 


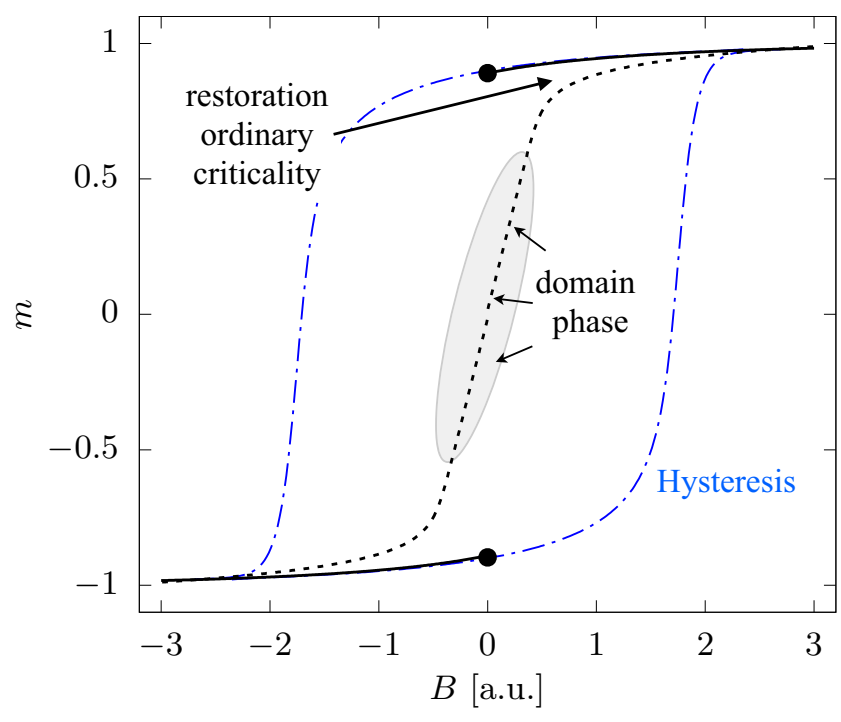

FIG. 1. Magnetization $m$ normalized to its saturation value as a function of $B$. The solid and dashed lines represent the equilibrium curves in the absence and in the presence of dipolar interaction, respectively. The two bullets highlight the nonanalyticity in $B=0$ expected only in the first case (see main text). The ellipse highlights the portion of the dashed curve corresponding to the multidomain phase, while the arrow indicates the region where standard criticality ( $M \sim B^{1 / \delta}$ for $T \simeq T_{\mathrm{c}}$ ) is restored. The dot-dashed (blue) curve represents a prototypical hysteresis: both equilibrium scenariossolid and dashed curves—are compatible with hysteresis.

magnetization curve of ferromagnets, is not an equilibrium phenomenon and is, therefore, beyond our scope. Consistent with the scenario depicted in the Introduction, the equilibrium magnetization for a real ferromagnet should behave smoothly as a function of the $B$ field when the latter passes from negative to positive values, without displaying any singularity at any temperature. The experimental validation of this fact is usually precluded because magnets are normally not able to relax to the configuration of minimal free energy within the measurement time. This means that magnetic hysteresis is also compatible with the vanishing of spontaneous magnetization at equilibrium prescribed by the Griffith's theorem, as a result of dipolar frustration. Together with other coworkers, we recently reported an experimental study in which the magnetization of a ferromagnet strongly frustrated by dipolar interaction was measured in a wide range of temperature $(T)$ and applied $B$ field, taking care that hysteretic effects were negligible [8]. This study was performed on Fe films epitaxially grown on $\mathrm{Cu}$. For a thickness smaller than three atomic $\mathrm{Fe}$ layers, these films are magnetized out of plane. In this configuration, the frustrating effect of dipolar interaction against ferromagnetism is maximal because the dipolar interaction between any pair of magnetic moments in the film is antiferromagnetic. As a reference model for those Fe films, we consider a 2D Ising Hamiltonian in which the usual nearest-neighbor ferromagnetic exchange interaction competes with an antiferromagnetic interaction decaying with the third power of the distance. This second term arises from the isotropic contribution to pairwise dipolar coupling, the anisotropic contribution vanishing exactly in films magnetized out of plane. The model Hamiltonian thus reads

$$
H=-J \sum_{\langle i, j\rangle} S_{i} S_{j}+g \sum_{i \neq j} \frac{S_{i} S_{j}}{r_{i j}^{3}}-h \sum_{i} S_{i},
$$

where $S_{i}= \pm 1$ are Ising spins disposed on a square lattice, representing the two out-of-plane directions along which magnetic moments preferentially point. The first sum runs over every distinct pair of nearest-neighboring sites (positive exchange coupling constant $J>0$ is assumed henceforth). The second sum, associated with dipolar interaction with strength $g>0$, runs now over every distinct pair of sites in the lattice. The last term represents the Zeeman energy $h=\mu B$, with $\mu$ magnetic moment. As a result of the competition between the short- and long-ranged interaction, this model exhibits modulated phases at low temperatures: striped phases at zero or small magnetic fields $[9,10]$ and bubble phases at intermediate fields [11-14]. Due to this feature this model and its generalized versions, in which the antiferromangetic coupling decays with a generic exponent $\alpha$, have been studied extensively during the last two decades in relation to the type of order realized in the modulated phases (smectic, Ising nematic, etc.) or to the possibility of producing self-generated glassiness [15-17]. The uniform phase can be enforced by applying a magnetic field larger than a certain threshold value $h_{\mathrm{c}}$, which is generally temperature dependent $[11,18]$. Whether some trace of the critical behavior, characterizing not frustrated models of ferromagnetism, is found in this uniform phase has eluded scientific interest so far. We remark that in the frustrated model the global magnetization is not an order parameter in the conventional understanding of second-order phase transitions. It is therefore a priori not obvious whether some critical behavior should be displayed at all in the uniform phase obtained when a large enough field is applied. The experiments on $\mathrm{Fe}$ films on $\mathrm{Cu}$ confirmed without any doubt that ordinary criticality indeed occurs in the uniform phase of a strongly frustrated ferromagnet. In that specific system, the scaling behavior of the magnetization,

$$
m(\tau, B)=|\tau|^{\beta} F^{ \pm}\left(\frac{B}{|\tau|^{\beta \delta}}\right)
$$

( $\tau=T / T_{\mathrm{c}}-1$ ), is realized when external fields larger than a certain temperature-dependent threshold $\left(B_{\mathrm{c}}\right)$ are applied and is consistent with the critical exponents $(\beta, \delta)$ and scaling functions of the (unfrustrated) 2D Ising model. The threshold field $B_{\mathrm{c}}$ varies from few Gauss for $\tau<-0.05$ to about 50 Gauss $\left(5 \times 10^{-3} \mathrm{~T}\right)$ around $\left.T_{\mathrm{c}}\right)$. In the-so-called-GriffithsWidom representation power laws are obeyed up to 80 orders of magnitude [8]. The essence of the experimental observations on $\mathrm{Fe}$ films on $\mathrm{Cu}$ was confirmed by Monte Carlo simulations performed using the Hamiltonian Eq. (1), even if realistic values of the Hamiltonian parameters could not be employed. For instance, using a physical value for the ratio $J / g(\sim 500)$ would produce magnetic domains of size larger than the accessible simulation boxes. Therefore, the much smaller ratio $\mathrm{J} / \mathrm{g}=10$ was used to observe both uniform and modulated phases in the same simulation. In experiments, the fields that suffice to stabilize the uniform phase correspond to a Zeeman energy of the order of $10^{-5}$ times $J$, while for the parameters used in Monte Carlo simulations these 
fields are about $10^{-1}-10^{-2}$ times $J$ (see later on). Differently from what done in the analysis of experimental results, the critical exponents of the 2D Ising model were assumed in the analysis of Monte Carlo results presented in Ref. [8]. Therein, the scaling behavior Eq. (2) was verified adjusting the putative critical temperature $T_{\mathrm{c}}$ to obtain the maximal collapsing of simulated data. The critical temperature deduced in this way showed a linear dependence on the strength of dipolar coupling $g$ :

$$
T_{\mathrm{c}, g}=T_{\mathrm{c}, 0}-11.3 \mathrm{~g}
$$

where $T_{\mathrm{c}, 0}=2 J /(\ln (1+\sqrt{2})) \approx 2.269 J$ is the Onsager critical temperature.

These experimental and numerical facts suggest that the scaling hypothesis reported in textbooks of magnetism should be phrased in more general terms.

\section{THE SCALING HYPOTHESIS}

Both experimental and numerical evidence indicates that the critical behavior outside the multidomain phase is controlled by the Onsager critical point, i.e., the critical point of the unfrustrated ferromagnet $(g=0)$, when $g / J \ll 1$. This fact provides a valuable hint to set the scaling hypothesis Eq. (2) in a broader framework. Concretely, it suggests replacing the scaling function $F^{ \pm}(x)$, which depends on a single variable, by a two-variable scaling function $G^{ \pm}(x, y)$. This allows accounting for an additional scaling field, parametrized by the variable $u=g / J$; henceforth, that acts as a relevant field for the unfrustrated critical point $\left(T=T_{\mathrm{c}, 0}, B=0\right)$, in the sense of renormalization group. Hence, we might assume that the magnetization $m(\tau, b, u)$ is a generalized homogeneous function satisfying the relation

$$
m\left(\lambda^{1 / \beta} \tau, \lambda^{\delta} b, \lambda^{1 / \omega} u\right)=\lambda m(\tau, b, u) \quad \forall \lambda \neq 0,
$$

where $b=h / J=\mu B / J$ and $\omega$ is a new critical exponent. When $u=0$ we recover the behavior Eq. (2) from Eq. (4). Choosing $\lambda^{1 / \omega} u=1$, we obtain the scaling form

$$
m(\tau, b, u)=u^{\omega} G^{ \pm}\left(\frac{|\tau|}{u^{\omega / \beta}}, \frac{b}{u^{\omega \delta}}\right),
$$

where \pm refers to $\tau>0$ or $\tau<0$, respectively. The scaling functions $G^{ \pm}(x, y)$ must satisfy some particular asymptotic behaviors. For instance, when $u \neq 0$ and $b \rightarrow 0$ the magnetization should vanish for any value of the temperature. From this follows the requirement

$$
\lim _{y \rightarrow 0} G^{ \pm}(x, y)=0 .
$$

Moreover, Eq. (2) must be recovered in the limit $u \rightarrow 0$ with $B$ finite, meaning that

$$
G^{ \pm}(x, y) \sim x^{\beta} F^{ \pm}\left(\frac{y}{x^{\beta \delta}}\right)
$$

for $x \gg 1$ and $y \gg 1$ with $y / x^{\beta \delta}$ finite. We then expect two different scaling regimes depending on whether

$$
\begin{aligned}
& b \ll b_{\mathrm{c}} \quad \Rightarrow \text { multidomain phase, } \\
& b \gg b_{\mathrm{c}} \quad \Rightarrow \text { ferromagnetic scaling region, }
\end{aligned}
$$

the crossover field scaling with $u$ as $b_{\mathrm{c}}=h_{\mathrm{c}} / J \propto u^{\omega \delta}$. In the first regime the equilibrium magnetization is a smooth function (essentially a straight line for Fe films on $\mathrm{Cu}$ ) of the applied field. In the second regime $\left(b \gg b_{c}\right)$ standard criticality expressed by Eq. (2) holds. Defining $p(y) \equiv G^{+}(0, y)=$ $G^{-}(0, y)$, for $\tau=0$ one has

$$
\frac{m}{u^{\omega}}=p\left(\frac{b}{u^{\omega \delta}}\right) .
$$

Finally, to be compatible with Eq. (4) the singular part of the free energy must also be a generalized homogeneous function and satisfy the relation

$$
f\left(\lambda^{\frac{1}{\beta(1+\delta)}} \tau, \lambda^{\frac{\delta}{1+\delta}} b, \lambda^{\frac{1}{\omega(1+\delta)}} u\right)=\lambda f(\tau, b, u) \quad \forall \lambda \neq 0 .
$$

In the next sections the validity of the scaling ansatz proposed in Eq. (4) will be confirmed studying the model Eq. (1) by means of different approaches: mean-field approximation, real-space renormalization group, Monte Carlo simulations.

\section{MEAN-FIELD THEORY}

To analyze the mean-field approximation of Hamiltonian Eq. (1), we consider the Landau-Ginzburg (LG) free energy

$$
\begin{aligned}
F[\phi]= & \frac{1}{2} \int d^{2} \mathbf{x}\left\{(J \nabla \phi(\mathbf{x}))^{2}+r_{0} \phi^{2}(\mathbf{x})+\frac{\bar{\lambda}}{2} \phi^{4}(\mathbf{x})\right\} \\
& +\frac{g}{2} \int d^{2} \mathbf{x} \int d^{2} \mathbf{x}^{\prime} \frac{\phi(\mathbf{x}) \phi\left(\mathbf{x}^{\prime}\right)}{\left|\mathbf{x}-\mathbf{x}^{\prime}\right|^{3}}-h \int d^{2} \mathbf{x} \phi(\mathbf{x}),
\end{aligned}
$$

where the scalar field $\phi(\mathbf{x})$ represents the out-of-plane spin density in a magnetic thin film with easy axis perpendicular to the film plane. Space variables $\mathbf{x}$ and $\mathbf{x}^{\prime}$ are assumed to be dimensionless so that the constants $J, r_{0}, \bar{\lambda}$, and $h$ have the units of an energy. The terms between curly brackets model the ferromagnetic exchange interactions in the continuum limit. In the vicinity of the critical point one has

$$
\begin{aligned}
& r_{0}=T-T_{\mathrm{c}, 0}^{\mathrm{MF}}, \\
& \bar{\lambda}=\frac{1}{3} T_{\mathrm{c}, 0}^{\mathrm{MF}},
\end{aligned}
$$

where $T_{\mathrm{c}, 0}^{\mathrm{MF}}$ is the mean-field transition temperature of the unfrustrated model (with $g=0$ ), for which the critical exponents are well-known: $\beta=1 / 2$ and $\delta=3$. Outside a limited region in the $(\tau, h)$ parameters space, i.e., for $h>h_{\mathrm{c}}$, domain states are not stable (they are either metastable or unstable) and the uniform solution $\phi=\phi_{0}$ is the equilibrium one [13]. The saddle-point equation for $\phi_{0}$ is

$$
\left(r_{0}+a_{g} g\right) \phi_{0}+\phi_{0}^{3}=h,
$$

where

$$
a_{g}=\int \frac{d^{2} \mathbf{x}}{|\mathbf{x}|^{3}} .
$$

One can implicitly assume a lower cutoff so that the integral above is not ill-defined. However, in the original functional Eq. (11) the dipolar interaction is well-defined in the domain of distributions. An explicit value can be assigned to the constant $a_{g}$ considering the coarse-grained (magnetostatic) description of the equivalent model, that is a slab of volume 
$V$ uniformly magnetized out of plane. For this system the demagnetizing energy is

$$
\mathcal{E}_{\mathrm{d}}=\frac{1}{2} \mu_{0} M^{2} V=\frac{1}{2} \mu_{0} \mu^{2}\left(\frac{\phi_{0}}{a^{3}}\right)^{2} V
$$

In the last equivalence a cubic lattice of constant $a$ has been assumed as well as the obvious relation $M=\mu \phi_{0} / a^{3}$ between the macroscopic magnetization $M$ and the uniform spin density $\phi_{0}$ considered here. The demagnetizing energy in Eq. (15) should equal the corresponding contribution in the LG functional

$$
\begin{aligned}
F_{\mathrm{d}}[\phi] & =\frac{g}{2} \int d^{2} \mathbf{x} \int d^{2} \mathbf{x}^{\prime} \frac{\phi(\mathbf{x}) \phi\left(\mathbf{x}^{\prime}\right)}{\left|\mathbf{x}-\mathbf{x}^{\prime}\right|^{3}} \\
& =\frac{1}{2} \frac{\mu_{0}}{4 \pi} \frac{\mu^{2}}{a^{3}} \phi_{0}^{2} \int d^{2} \mathbf{x}^{\prime} \int \frac{d^{2} \mathbf{x}}{|\mathbf{x}|^{3}},
\end{aligned}
$$

where in the second passage we have expressed the coupling constant $g$ in terms of the atomic magnetic moment and the lattice unit. A one-to-one mapping can now be established between individual terms in the Eqs. (15) and (16):

$$
\begin{aligned}
\frac{\mu}{a^{3}} \phi_{0} & =M, \\
\mu \phi_{0} \int d^{2} \mathbf{x}^{\prime} & =M V, \\
\frac{1}{4 \pi} \int \frac{d^{2} \mathbf{x}}{|\mathbf{x}|^{3}} & =1 .
\end{aligned}
$$

Therefore, in this description, the constant in Eq. (14) is $a_{g}=4 \pi$. From the saddle-point Eq. (13) associated with a uniform spin density $\phi_{0}$, we note that the presence of dipolar interaction effectively lowers the critical temperature by an amount $4 \pi g$, namely,

$$
T_{\mathrm{c}, g}^{\mathrm{MF}}=T_{\mathrm{c}, 0}^{\mathrm{MF}}-4 \pi g .
$$

Even if deduced in a mean-field context, the correction to the critical temperature provided by dipolar coupling is in excellent agreement with the results of Monte Carlo simulations on a square lattice summarized in Eq. (3).

Let us go back to the main purpose of this section of verifying the validity of our scaling hypothesis within the mean-field approximation. To this aim, we define $z=\phi_{0} / u^{1 / 2}$ and divide both sides of the saddle-point Eq. (13) by $u^{3 / 2}$ to obtain

$$
\left(a_{0} \frac{\tau}{u}+a_{1}\right) z+z^{3}=\frac{h}{u^{3 / 2}},
$$

where $a_{0}$ and $a_{1}$ are numerical constants. Therefore, we obtain

$$
\phi_{0}=u^{1 / 2} G^{ \pm}\left(\frac{|\tau|}{u}, \frac{h}{u^{3 / 2}}\right),
$$

which is consistent with the scaling hypothesis proposed in Eq. (5), with a mean-field dipolar critical exponent $\omega=1 / 2$ characterizing the dipolar relevant field $u$.

\section{REAL-SPACE RENORMALIZATION GROUP APPROACH (RSRG)}

A fundamental requirement for the consistency of the scaling hypothesis in Eq. (4) is that the variable $u$ be a relevant field within a renormalization-group (RG) approach. In the following we demonstrate that this is indeed the case using the Niejmeijer and van Leeuwen RSRG technique [19] and its extension to include long-range interactions [20]. For the sake of simplicity, we prove this only for the $B=0$ case, the extension to $B \neq 0$ being straightforward. Defining $\mathcal{H} \equiv$ $-\beta H$, from Eq. (1) we have

$$
\mathcal{H}=K_{1} \sum_{\langle i, j\rangle} S_{i} S_{j}-K_{2} \sum_{i \neq j} \frac{S_{i} S_{j}}{r_{i j}^{3}},
$$

namely, $K_{1}=\beta J$ and $K_{2}=\beta g$. We divide the system into Kadanoff blocks with $\Lambda$ spins, so that the rescaling length of the RG transformation is $l=\sqrt{\Lambda}$. To each block $I$ we assign a block spin $S_{I}^{\prime}= \pm 1$. Defining the renormalized block Hamiltonian as

$$
\mathcal{H}^{\prime}=K_{1}^{\prime} \sum_{\langle I, J\rangle} S_{I}^{\prime} S_{J}^{\prime}-K_{2}^{\prime} \sum_{I \neq J} \frac{S_{I}^{\prime} S_{J}^{\prime}}{r_{I J}^{3}}
$$

where $r_{I J}$ is the distance between blocks $I$ and $J$ measured in units of the rescaled length $l$, we obtained the recursion RG equations

$$
\begin{aligned}
& K_{1}^{\prime}=K_{1}^{\prime}\left(K_{1}, K_{2}\right), \\
& K_{2}^{\prime}=K_{1}^{\prime}\left(K_{1}, K_{2}\right),
\end{aligned}
$$

for each block set. In Fig. 2 we show the different Kadanoff blocks used in the RG calculation. The details of the RG implementation are given in the Appendix. It is immediate to see that $K_{2}=0$ implies $K_{2}^{\prime}=0$. Therefore, the nontrivial fixed point of the RG Eqs. (23) is located at $\left(K_{\mathrm{c}}, 0\right)$, with $K_{\mathrm{c}}$ determined by the equation $K_{1}^{\prime}\left(K_{\mathrm{c}}, 0\right)=K_{\mathrm{c}}$. In the RG approach, this fixed point corresponds to the critical temperature, i.e., $T_{\mathrm{c}, 0}^{\mathrm{RG}}=J / k_{B} K_{\mathrm{c}}$ is the transition temperature of the unfrustrated model under the present approximation. Still in zero magnetic field, the RG equations linearized around this critical point are given by the matrix

$$
\left(\begin{array}{ll}
\frac{\partial K_{1}^{\prime}}{\partial K_{1}} & \frac{\partial K_{1}^{\prime}}{\partial K_{2}} \\
\frac{\partial K_{2}^{\prime}}{\partial K_{1}} & \frac{\partial K_{2}^{\prime}}{\partial K_{2}}
\end{array}\right)_{K_{1}=K_{\mathrm{c}}, K_{2}=0}=\left(\begin{array}{ll}
\lambda_{\tau} & \frac{\partial K_{1}^{\prime}}{\partial K_{2}} \\
\frac{\partial K_{2}^{\prime}}{\partial K_{1}} & \frac{\partial K_{2}^{\prime}}{\partial K_{2}}
\end{array}\right)_{K_{1}=K_{\mathrm{c}}, K_{2}=0},
$$

where $\lambda_{\tau}$ is the so-called thermal eigenvalue (see the Appendix). From Eq. (A3) we have

$$
\left.\frac{\partial K_{2}^{\prime}}{\partial K_{1}}\right|_{K_{1}=K_{\mathrm{c}}, K_{2}=0}=0
$$

and, therefore, the eigenvalue associated with the dipolarcoupling constant (in units of $k_{\mathrm{B}} T$ ) $K_{2}$ is given by

$$
\lambda_{u}=\left.\frac{\partial K_{2}^{\prime}}{\partial K_{2}}\right|_{K_{1}=K_{\mathrm{c}}, K_{2}=0}=\frac{1}{l^{3}}\left[M\left(K_{\mathrm{c}}\right)\right]^{2},
$$

where $M(K)$ is given in Eq. (A6). It is also easy to see that the field eigenvalue is given by $\lambda_{b}=M\left(K_{\mathrm{c}}\right)$.

The main idea behind the present approach is that eigenvalues of the RG equations linearized around a nontrivial fixed point that are larger than one correspond to relevant fields $[2,4,7]$. The aim of this section is, thus, to prove that $\lambda_{u}>1$. Typically, eigenvalues associated with relevant fields display a power-law dependence on the size of the Kadanoff blocks that are specific of the implemented renormalization procedure 
(a)

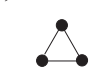

$\Lambda=3$

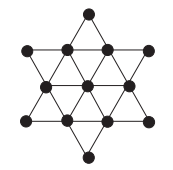

$\Lambda=13$

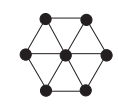

$\Lambda=7$

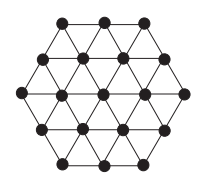

$\Lambda=19$

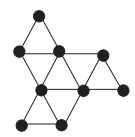

$\Lambda=9$

(b)
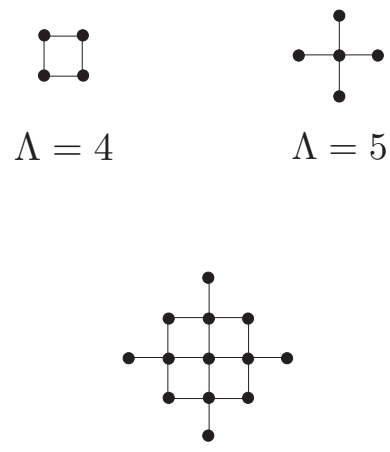

$\Lambda=13$

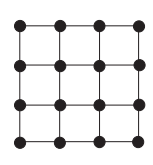

$\Lambda=16$

FIG. 2. Kadanoff blocks for different values of $\Lambda$ used in the RG approach for (a) triangular lattice and (b) square lattice.

[21]. The relative exponents are directly related to physical critical exponents (see below).

We calculated the eigenvalues for different values of $\Lambda$, both for the triangular and the square lattices shown in Fig. 2. Since the critical exponents are expected to be independent of the lattice structure, we could expect the general trend of the eigenvalues with $l$ to be the same for both lattices. We now make a change of variables from $\left(K_{1}, K_{2}\right)$ to $(\tau, u)$, with $u=K_{2} / K_{1}, \tau=K_{\mathrm{c}} / K_{1}-1$. Then, neglecting in first approximation the nondiagonal element of the RG matrix and assuming

$$
\lambda_{\tau} \sim l^{y_{\tau}}, \quad \lambda_{b} \sim l^{y_{b}}, \quad \lambda_{u} \sim l^{y_{u}}
$$

(where we have included now a finite magnetic field), it is easy to show that the singular part of the free energy should scale, close to the critical point, as

$$
f\left(l^{y_{\tau} / d} \tau, l^{y_{b} / d} b, l^{y_{u} / d} u\right) \approx l f(\tau, b, u),
$$

with $d=2$ being the dimensionality of the system. Hence, comparing the equation above with Eq. (10) one obtains that

$$
\begin{gathered}
y_{\tau}=\frac{2}{\beta(1+\delta)}=\frac{1}{v}, \\
y_{b}=\frac{2 \delta}{1+\delta}, \\
y_{u} \approx \frac{2}{\omega(1+\delta)} .
\end{gathered}
$$
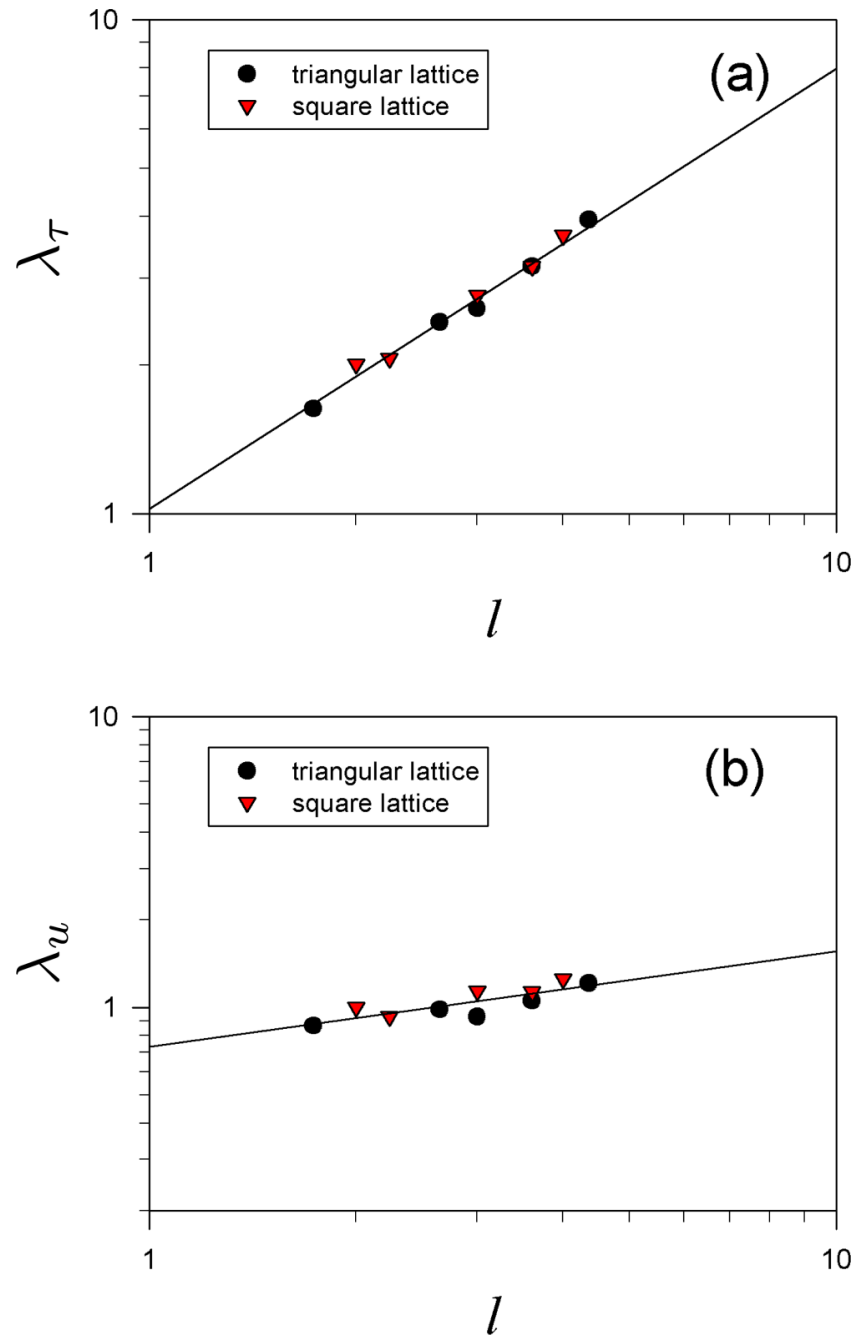

FIG. 3. Thermal and dipolar eigenvalues vs. the scale length $l$. (a) $\lambda_{\tau}$. (b) $\lambda_{u}$.

While the first two equations are well-known, to the best of our knowledge, the third Eq. (28) is not reported in the literature. This last equation allows relating the dipolar critical exponent $\omega$ to the other exponents.

As a consistency check of our RG approach, we first calculated $\lambda_{\tau}$ as a function of $l=\sqrt{\Lambda}$. Figure 3(a) shows that $\lambda_{\tau}$ displays the expected power-law behavior (line in a log-log scale). A linear fitting combining the data for both the square and the triangular lattice yields the exponent $y_{\tau}=0.89$, reasonably close to the exact value $y_{\tau}=1$. We then proceeded considering the behavior of the eigenvalue associated with the dipolar coupling, of our interest. Figure 3(b) shows $\lambda_{u}$ as a function of $l$. As one can see, $\lambda_{u}$ is actually smaller than one for small values of $l$, because $\lambda_{u}=\lambda_{0} l^{y_{u}}$ with $\lambda_{0}<$ 1. However, this eigenvalue also clearly obeys a power-law behavior, which indicates that $\lambda_{u}$ becomes larger than one when sufficiently large Kadanoff blocks are considered [20]. In this sense, the crucial result of this section is that $y_{u}>0$, which confirms the assumption of $u$ being a relevant field. The exponent resulting from the fit of the $\lambda_{u}$ eigenvalues computed for different lattices is $y_{u}=0.33$. From Eqs. (26)(28) this implies $\omega=1.015$. For completeness, it is worth 
mentioning that the same calculation for the field eigenvalue $\lambda_{b}$ yields a critical exponent $\delta=4.97$, very different from the exact result $\delta=15$. Hence, the value of $\omega$ resulting from this RG calculation can deviate significantly from the estimate of the same critical exponent obtained from Monte Carlo simulations.

We note that the nondiagonal structure of the RG matrix implies that the eigenvectors associated with the eigenvalues $\lambda_{\tau}$ and $\lambda_{u}$ are actually not orthogonal. Taking this into account [19] would produce a correction in the critical temperature $T_{\mathrm{c}, 0}^{\mathrm{RG}}=J / k_{B} K_{\mathrm{c}}$, which scales linearly with the strength of dipolar coupling $g$. Even without developing the calculation in details, the outcome would then be consistent with our Monte Carlo and mean-field results [see Eqs. (3) and (18)]. A second consequence of the nonorthogonality of eigenvectors is a correction in Eq. (28), which could lead to an improved estimate of the critical exponent $\omega$.

One may wonder whether a scaling ansatz similar to that of Eq. (4) could be used to characterize the crossover to the modulated phase realized for $b \ll b_{\mathrm{c}}$ [see Eq. (8)]. That would imply the existence of an associated nontrivial fixed point in the RG equations, as well as some attractor associated with the modulated ground state. We tried to explore this possibility within the present RG approach. We observed that, at least for the smallest cluster sizes (up to $\Lambda=5$, where the problem was easy tractable), the approach breaks down when one departs from the ferromagnetic region, because the RG equations become singular for large values of the dipolar coupling $g$. That behavior could be just a spurious effect of the present RSRG approach, but it could also indicate the absence of a simple second-order critical point. The second alternative suggests that there is no second-order phase transition at all in the modulated phase. Indeed, many theoretical works point in this direction, the most probable scenario being something similar to a Kosterlitz-Thouless phase transition [14,22].

\section{MONTE CARLO SIMULATIONS}

Monte Carlo simulations were performed using Hamiltonian Eq. (1) on a square lattice comprising $N=L \times L$ sites, with $L=200$ for all simulations; periodic boundary conditions were assumed and handled implementing Ewald sums. We calculated the total magnetization $m=\sum\left\langle S_{i}\right\rangle / N$ (where $\left\langle S_{i}\right\rangle$ stands for the statistical average) as a function of $h$ at $T=T_{\mathrm{c}, 0} \approx 2.27 \mathrm{~J}$ for $h>h_{\mathrm{c}}$ and different values of $u=g / J$. The critical field $h_{\mathrm{c}}$ was estimated, either by direct calculation of the corresponding order parameters (for small values of $J / g$ ) or by a zero-field cooling-field-cooling procedure, as described in Ref. [8]. At the beginning of each MC run we let the system equilibrate over 1000 Monte Carlo Steps (MCS) and then average over 1000 sampling point taken every 100 MCS along a single MC run.

In Fig. 4(a) we show the simulated magnetization curves as a function of $h$ for different values of $J / g$ computed at $T=T_{\mathrm{c}, 0}$. In Fig. 4(b) we show a scaling plot of $m / u^{\omega}$ vs. $h / u^{\omega \delta}$ for the same data set assuming $\omega \approx 1 / 10$ and $\delta=15$. The excellent collapsing of data confirms the scaling relation Eq. (9), which descends directly from the proposed scaling hypothesis Eq. (5).
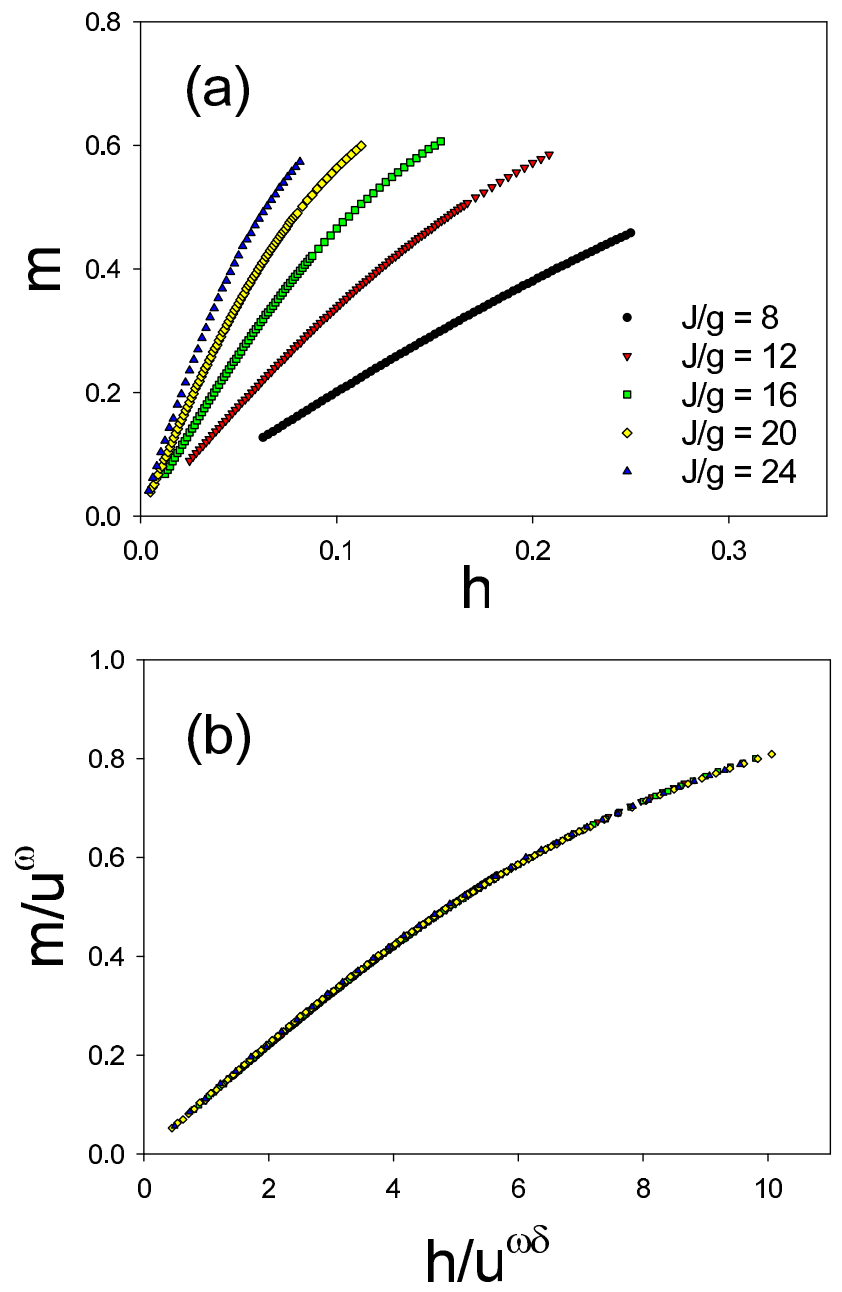

FIG. 4. Monte Carlo simulations for $L=200, T=T_{\mathrm{c}, 0}$ and different values of $J / g$. (a) Magnetization as a function of $h$. Data collapse of the same magnetization curves for $\omega=0.1 \pm 0.002$ $(\delta=15)$.

\section{DISCUSSION}

We proposed an extension of the textbook scaling ansatz for ferromagnets that applies to the realistic situation in which the formation of magnetic domains-promoted by dipolar interaction-renders the Curie point technically unreachable. This ansatz is based on the assumption that the dipolar coupling acts as a relevant field, in the sense of renormalization group. This implies that a dipolar critical exponent $\omega$ needs to be introduced, besides the traditional ones related to the ferromagnetic-to-paramagnetic phase transition. The most reliable estimate of this exponent is the one resulting from Monte Carlo simulations, i.e., $\omega \approx 1 / 10$. In fact, in this respect, the accuracy of mean-field theory and real-space renormalization-group approach is notoriously poor even for the unfrustrated model [4,21]. However, both these analytic approaches support the basic assumption of dipolar coupling being a relevant field.

Since dipolar interactions are ubiquitous and unavoidable in real magnets, our results suggest that long-range ferromagnetic order should be regarded as a crossover phenomenon. In other words, in the realm of equilibrium thermodynamics, 
the scaling behavior associated with the onset of long-range ferromagnetic ordering should be observable in the neighborhood of the putative critical point, but not too close to it. In fact, when the latter is approached by letting all the relevant fields $(\tau, b, u)$ go to zero, phases with modulated magnetization intervene that display a nonsingular behavior of the ferromagnetic order parameter $(m)$. In this perspective, our results reconcile the Griffith's theorem [5,6] and dipolar frustration with the observation of criticality, at least for ferromagnetic films magnetized out of plane.

Although the present work is only directly applicable to such films, where only the isotropic part of the dipolar interaction acts, we hope that it will stimulate further investigations aimed at validating the proposed scaling hypothesis Eq. (5) for 3D magnets.

In a broader perspective, the model studied by us is a special case of a class of models in which a generic exponent $\alpha$ is assumed for the power decay of the long-range interaction [14,23-25]. Beside the case with $\alpha=3$, another interaction that possesses an experimental counterpart is the Coulomb interaction $(\alpha=1)$, for which the model is called Coulombfrustrated ferromagnet. In both cases, with $\alpha=3$ and $\alpha=1$, the competition between the long-ranged antiferromagnetic interaction and the short-ranged ferromagnetic interaction leads to the formation of a modulated ground state, whose elementary excitations are described by an elastic-like Hamiltonian associated with the displacement of domain walls. In the limit in which the coupling of the long-ranged interaction (our $g$ ) is much smaller than $J$, such excitations are gapless, implying that the modulated phase lacks positional order at finite temperature for $2 \mathrm{D}$ systems $[14,22,26]$. This suggests that not even a kind of staggered magnetization may display 2DIsing critical behavior observed, instead, when the uniform phase is enforced by an external field (main result of Ref. [8]). The thermodynamics of this class of models is dictated by the temperature dependence of multiple characteristic length scales [16,27]. To the best of our knowledge, only the period of modulation $L$ has been studied extensively in ferromagnetic films $[11,18,28,29]$. In particular, the relation $m / h \sim L$ was conjectured to hold for the modulated phase and supported by experimental results in Ref. [8]. Therein, this fact was used to mark a separatrix between the region in which the magnetic susceptibility of Fe films depended smoothly on $T$ (modulated phase) and where it obeyed a critical behavior (uniform phase). Distinguishing between these two regimes was crucial to estimate the $\delta$ critical exponent experimentally. Indeed, this separatrix is the analog of the "disorder line" introduced Ref. [27]: when crossing such a disorder line no phase transition occurs but observables, like the susceptibility, may display a crossover. As anticipated, in these models the presence of multiple temperature-dependent length scales is expected to trigger self-generated glassiness [15-17] and avoided criticality. The second wording is used in situations in which a second-order critical point occurring in the absence of frustration is replaced by a modulated phase as soon as an infinitesimal amount of (power-law decaying) frustration is considered [30,31]. The Coulomb-frustrated ferromagnet [37] was originally proposed to study charged stripes in cuprate high- $T_{\mathrm{c}}$ superconductors [30,32]. A nonexhaustive list of other pattern-forming systems for which the phenomenology of avoided criticality has been proposed comprises magnetic films, ferrofluids, diblock copolymers, amphiphilic solutions, systems undergoing Turing-like phase separating chemical reactions [31,33-36]. The approach presented here could potentially help understand the complex phase diagram of all these physical systems.

\section{ACKNOWLEDGMENTS}

This work was partially supported by CONICET (Argentina) through Grant No. PIP 11220150100285 and SeCyT (Universidad Nacional de Córdoba, Argentina). We thank S. Bustingorry and T. Grigera for fruitful suggestions. Danilo Pescia is acknowledged for putting this interesting open problem under our attention and for several illuminating discussions we had with him on this topic.

\section{APPENDIX: REAL-SPACE RENORMALIZATION GROUP IMPLEMENTATION}

Following Niejmeijer and van Leeuwen prescription, we first divided the Hamiltonian Eq. (21) into two parts: $\mathcal{H}=$ $\mathcal{H}_{0}+\mathcal{V}$, where $\mathcal{H}_{0}=\sum_{I} \mathcal{H}_{0}^{I}$ and $\mathcal{V}=\sum_{I \neq J} \mathcal{V}_{I J} ; \mathcal{H}_{0}^{I}$ includes only the interactions between spins inside the block $I$, whereas $\mathcal{V}_{I J}$ includes the interactions between spins belonging to different blocks $I$ and $J$. We also denoted $S_{i}^{I}$ $(i=1, \ldots, \Lambda)$ the site spins belonging to the block $I$. The renormalized Hamiltonian Eq. (22), in the first order cumulant approximation $[19,20]$ is then given by

$$
\mathcal{H}^{\prime}=\sum_{I \neq J}\left\langle\mathcal{V}_{I J}\right\rangle_{0}
$$

where

$$
\langle\mathcal{O}\rangle_{0}=\frac{1}{Z_{0}} \operatorname{Tr}_{\left\{S_{i}^{I}\right\}} P\left(\left\{S_{i}^{I}\right\},\left\{S_{I}^{\prime}\right\}\right) \exp \left[\mathcal{H}_{0}\left(\left\{S_{i}^{I}\right\}\right)\right] \mathcal{O}
$$

with

$$
Z_{0}=\operatorname{Tr}_{\left\{S_{i}^{I}\right\}} P\left(\left\{S_{i}^{I}\right\},\left\{S_{I}^{\prime}\right\}\right) \exp \left[\mathcal{H}_{0}\left(\left\{S_{i}^{I}\right\}\right)\right]
$$

and

$$
P\left(\left\{S_{i}^{I}\right\},\left\{S_{I}^{\prime}\right\}\right)=\prod_{I} \frac{1}{2}\left[1+S_{I}^{\prime} \operatorname{sgn}\left(\sum_{i=1}^{\Lambda} S_{i}^{I}\right)\right],
$$

is the weight function which characterizes the majority rule recipes. We will use this expression also when $\Lambda$ is an even number, meaning that $P$ assigns the values $S_{I}^{\prime}= \pm 1$ with probability $1 / 2$ to spins configurations with zero magnetization in the block. In particular, it is easy to see that $\left\langle S_{i}^{I}\right\rangle_{0}=a_{i}(\vec{K}) S_{I}^{\prime}$, where $a_{i}(\vec{K})$ does not depend on the block $I$. Assuming now that [20] $r_{i j} \approx l r_{I J}$ for $r_{I J}>1$, replacing into Eqs. (21) and (A1), and comparing with Eq. (22), after some straightforward algebra we find

$$
\begin{gathered}
K_{2}^{\prime}=\frac{K_{2}}{l^{3}}\left[\sum_{i \in I} a_{i}(\vec{K})\right]^{2}, \\
K_{1}^{\prime}=K_{1} \sum_{i \in I}^{\prime} \sum_{j \in J}^{\prime} a_{i} a_{j}-K_{2} \sum_{i \in I} \sum_{j \in J} \frac{1}{r_{i j}^{3}} a_{i} a_{j}+K_{2}^{\prime},
\end{gathered}
$$


where $I$ and $J$ in the last equation are nearest-neighboring blocks. The first pair of sums (primed sums) in Eq. (A4) run over nearest-neighboring sites $i$ and $j$, while the second pair run over all sites in both blocks. Other useful block-dependent quantities were calculated, such as

$$
\left.L(K) \equiv \sum_{i \in I}^{\prime} \sum_{j \in J}^{\prime} a_{i} a_{j}\right|_{K_{1}=K, K_{2}=0}
$$

and

$$
\left.M(K) \equiv \sum_{i \in I} a_{i}(\vec{K})\right|_{K_{1}=K, K_{2}=0} .
$$

For instance, the ferromagnetic (short range) critical point is determined by $L\left(K_{\mathrm{c}}\right)=1$ and the corresponding thermal eigenvalue $\lambda_{\tau}=l^{1 / \nu}$ by

$$
\lambda_{\tau}=\left.\frac{\partial K_{1}^{\prime}}{\partial K_{1}}\right|_{K_{1}=K_{\mathrm{c}}, K_{2}=0}=1+\left.K_{\mathrm{c}} \frac{d L}{d K}\right|_{K=K_{\mathrm{c}}} .
$$

We see that, to determine the stability of the ferromagnetic fixed point under the present approximation we just need the quantities

$$
a_{i}^{\prime}(K)=a_{i}(K, 0)=\left\langle S_{i}^{I}\right\rangle_{0} \mid S_{I}^{\prime}=1, K_{1}=K, K_{2}=0 .
$$

Let us consider a simple example for $\Lambda=5$, which corresponds to a cross-shaped Kadanoff block [see Fig. 2(b)]. Suppose that we label $i=0$ the central site and $i=1,2,3,4$ the external sites of the block. By symmetry, the coefficients $a_{i}^{\prime}(K)=a_{e}(K), i=1,2,3,4$, are all equivalent. Then from Eqs. (A2) and (A8) we obtain

$$
a_{e}(K)=\frac{e^{4 K}+e^{-4 K}+2 e^{2 K}+2 e^{-2 K}}{6+e^{4 K}+e^{-4 K}+4 e^{2 K}+4 e^{-2 K}}
$$

and for the central site

$$
a_{0}^{\prime}(K)=\frac{6+e^{4 K}-e^{-4 K}+4 e^{2 K}-4 e^{-2 K}}{6+e^{4 K}+e^{-4 K}+4 e^{2 K}+4 e^{-2 K}} .
$$

Between two neighboring blocks there are three firstneighbor bonds. Hence, $L(K)=3\left[a_{e}(K)\right]^{2}$ and $M(K)=$ $4 a_{e}(K)+a_{0}(K)$. For large clusters the functions $a_{i}(K)$ can be obtained with the aid of symbolic manipulation programs, for clusters of size up to $\Lambda \approx 20$ (see Fig. 2).
[1] A. Taroni, S. T. Bramwell, and P. C. W. Holdsworth, J. Phys.: Condens. Matter 20, 275233 (2008).

[2] H. E. Stanley, Rev. Mod. Phys. 71, S358 (1999).

[3] N. Goldenfeld, Lectures on Phase Transitions and the Renormalization Group, Vol. 85 of Frontiers in Physics (Perseus Books Publishing, Reading, Massachusetts, 1992).

[4] M. Le Bellac, Quantum and Statistical Field Theory, Oxford Science Publishing (Clarendon Press, Oxford, 1991).

[5] R. B. Griffiths, Phys. Rev. 176, 655 (1968).

[6] A. Campa, T. Dauxois, D. Fanelli, and S. Ruffo, Physics of Long-Range Interacting Systems (Oxford University Press, Oxford, 2014).

[7] M. E. Fisher, Rev. Mod. Phys. 46, 597 (1974).

[8] N. Saratz, D. A. Zanin, U. Ramsperger, S. A. Cannas, D. Pescia, and A. Vindigni, Nat. Commun. 7, 13611 (2016).

[9] S. A. Pighin and S. A. Cannas, Phys. Rev. B 75, 224433 (2007).

[10] A. Vindigni, N. Saratz, O. Portmann, D. Pescia, and P. Politi, Phys. Rev. B 77, 092414 (2008).

[11] O. Portmann, A. Gölzer, N. Saratz, O. V. Billoni, D. Pescia, and A. Vindigni, Phys. Rev. B 82, 184409 (2010).

[12] R. Diaz-Mendez and R. Mulet, Phys. Rev. B 81, 184420 (2010).

[13] S. A. Cannas, M. Carubelli, O. V. Billoni, and D. A. Stariolo, Phys. Rev. B 84, 014404 (2011).

[14] A. Mendoza-Coto, D. A. Stariolo, and L. Nicolao, Phys. Rev. Lett. 114, 116101 (2015).

[15] J. Schmalian and P. G. Wolynes, Phys. Rev. Lett. 85, 836 (2000).

[16] Z. Nussinov, Phys. Rev. B 69, 014208 (2004).

[17] A. Principi and M. I. Katsnelson, Phys. Rev. Lett. 117, 137201 (2016).

[18] N. Saratz, A. Lichtenberger, O. Portmann, U. Ramsperger, A. Vindigni, and D. Pescia, Phys. Rev. Lett. 104, 077203 (2010).

[19] T. Niemeijer and J. M. J. van Leeuwen, Phys. Rev. Lett. 31, 1411 (1973).

[20] S. A. Cannas, Phys. Rev. B 52, 3034 (1995).

[21] B. Delamotte, in Renormalization Group and Effective Field Theory Approaches to Many-Body Systems, edited by J. Polonyi and A. Schwenk (Springer-Verlag, Berlin, 2012).

[22] D. G. Barci and D. A. Stariolo, Phys. Rev. B 79, 075437 (2009).

[23] A. Giuliani, J. L. Lebowitz, and E. H. Lieb, Phys. Rev. B 84, 064205 (2011).

[24] A. Giuliani, J. L. Lebowitz, and E. H. Lieb, Phys. Rev. B 76, 184426 (2007).

[25] M. Grousson, G. Tarjus, and P. Viot, Phys. Rev. E 62, 7781 (2000).

[26] S. A. Cannas, D. A. Stariolo, and F. A. Tamarit, Phys. Rev. B 69, 092409 (2004).

[27] Z. Nussinov, J. Rudnick, S. A. Kivelson, and L. N. Chayes, Phys. Rev. Lett. 83, 472 (1999).

[28] M. Kronseder, M. Buchner, H. G. Bauer, and C. H. Back, Nat. Commun. 4, 2054 (2013).

[29] M. Kronseder, T. N. G. Meier, M. Zimmermann, M. Buchner, M. Vogel, and C. H. Back, Nat. Commun. 6, 6832 (2015).

[30] V. J. Emery and S. A. Kivelson, Physica C 209, 597 (1993).

[31] S. A. Kivelson, E. Fradkin, and V. J. Emery, Nature 393, 550 (1998).

[32] J. M. Tranquada, B. J. Sternlieb, J. D. Axe, Y. Nakamura, and S. Uchida, Nature 375, 561 (1995).

[33] S. Chakrabarty, V. Dobrosavljević, A. Seidel, and Z. Nussinov, Phys. Rev. E 86, 041132 (2012).

[34] M. Seul and D. Andelman, Science 267, 476 (1995).

[35] D. Andelman and R. E. Rosensweig, J. Phys. Chem. B 113, 3785 (2009).

[36] B. Keimer, S. A. Kivelson, M. R. Norman, S. Uchida, and J. Zaanen, Nature 518, 179 (2015).

[37] In this toy model for high- $T_{\mathrm{c}}$ superconductors the short-range attraction among holes is assumed to arise from the presence of an underlying antiferromagnetic phase. The competition between this attractive interaction and Coulomb repulsion then produces hole-rich regions alternating with hole-poor antiferromagnetic regions within a sample, equivalent to magnetic domains in dipolar-frustrated model $[30,36]$. 